The 49th Annual Conference of the International Association of School Librarianship

The 24th International Forum on Research on School Librarianship

July $12-16,2021$

Kanae Suzuki, Hana Suzuki

University of Tsukuba, Japan, University of Tsukuba, Japan

kanae@slis.tsukuba.ac.jp; suzuki.hana.xd@alumni.tsukuba.ac.jp

\title{
Development and Evaluation of An Investigative Learning Support Program for Elementary School Students to Search and Understand Subjects in Social Studies from Multiple Dimensions
}

\begin{abstract}
This study developed an investigative learning support program that had two steps with multiple features including pillars of investigation for searching and grasping subjects of learning from multiple dimensions and then evaluated the goal achievement of the program. We examined literature and included reference materials and image maps, both with and without clues, and incorporated the tasks of revising or adding pillars of investigation in the program. Although there are some issues such as the consideration of instructions and prior support methods for students who have little experience in investigative learning, some students met the achievement goals of the program.
\end{abstract}

Keywords: investigative learning, Japan

\section{Introduction}

With the progress of globalization, deep independent and interactive learning has been promoted in Japan's Courses of Study, as established by MEXT (Ministry of Education, Culture, Sports, Science and Technology, 2017). Investigative learning, an example of such learning, is practiced to broadly expand students' conceptual knowledge, which is the subject of investigative learning in many subjects, including social studies. How to support investigative learning is an essential issue.

\section{Literature Review}

There are various definitions of investigative learning. Kuwata (2016) suggests two features as differences between investigative learning and inquiry-based learning. The first is the setting of subjects. In most cases with investigative learning, learners are given a subject in common to tackle, while in inquiry-based learning, the individual selection of subjects is located in the stage of subject setting. The second difference is in how learners summarize the results of their work. Investigative learning is typically closed-ended, with learners discovering the answer to a question, while inquiry-based learning is open-ended and allows for the possibility that no clear answer will be found. 
In each teaching unit, conceptual knowledge (e.g., characteristics of land topography) comprises multiple units of concrete knowledge (e.g., our land comprises large and small islands surrounded by the sea) (Tokyo Shoseki, 2015). It has a hierarchical knowledge structure in which the concrete knowledge is composed of terms and phrases (e.g., mountains, highlands, plateaus, hills, flatlands, plains, and basins).

Concrete knowledge includes knowledge from several similar fields, such as "three-quarters of Japan's land is mountainous," "less flat land," and "rivers flow more quickly in Japan than in other countries." The conceptual knowledge, shown in Figure 1, is composed of three different dimensions: "Japan's land is mountainous and has few plains (concrete knowledge A) and is composed of large and small islands surrounded by the sea (concrete knowledge B). In addition, there are many volcanoes, and hot springs are used for tourism, rest, power generation, etc. (concrete knowledge C)." Each piece of concrete knowledge contains multiple similar items of knowledge: three-quarters of the land is mountainous (concrete knowledge A-1), and there is little flat land (concrete knowledge A-2).

\section{Figure 1}

\section{Structure of Knowledge}

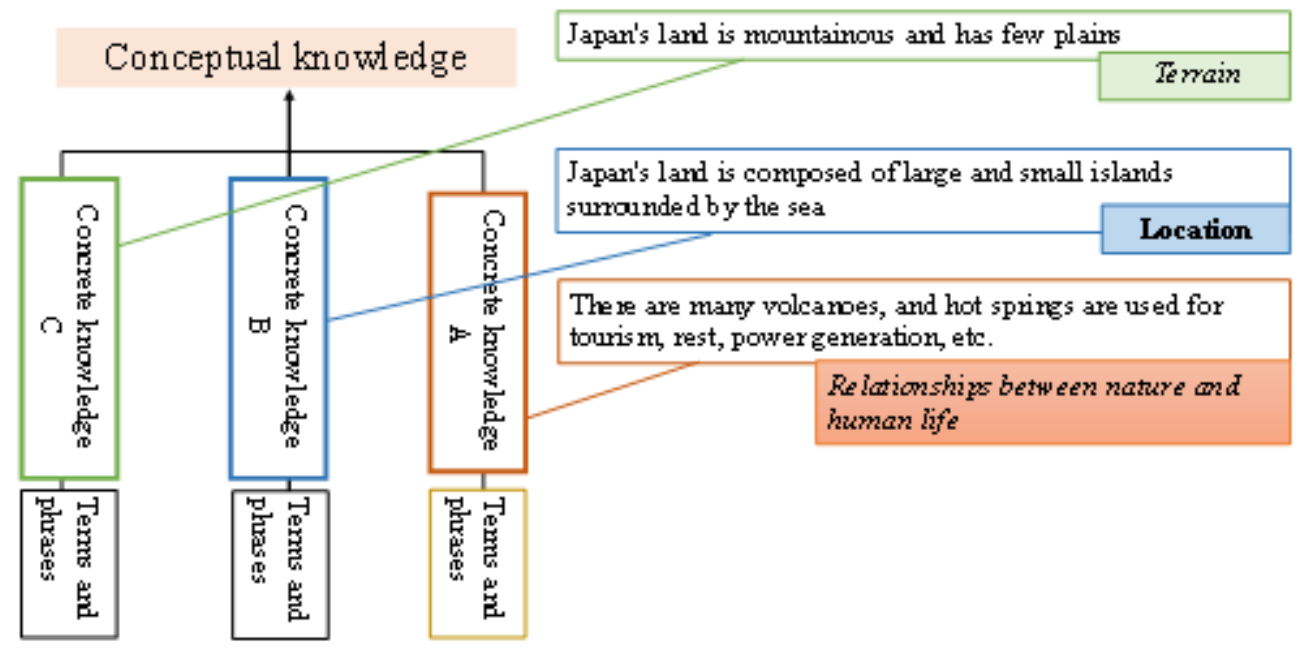

Note. Figure 1 is based on Tokyo Shoseki (2015).

As a generalized process of investigative learning, Tokuda (2009) suggested the following six stages: making a plan (deciding a sub-subject), gathering information, searching based on the gathered information, solving a problem, creating presentation materials, and presentation. 
These stages can be combined into three stages: planning, gathering information, and summary including searching based on the gathered information, solving a problem.

To broadly understand conceptual knowledge, it is necessary to acquire more concrete knowledge with different content. Thus, it is important to establish various dimensions (pillars of investigation) before searching for information. Learners often encounter difficulties during this planning stage (Kuhlthau, et. al., 2015).

Therefore, to broadly acquire conceptual knowledge, which is the subject of investigative learning, it is not enough to acquire only a plurality of similar concrete knowledge (e.g., concrete knowledge A-1 and A-2). It is necessary for learners to acquire more concrete knowledge (sub-subjects) with different content.

Engaging with deep independent and interactive learning is desirable in every subject. According to Japan's Courses of Study, basic/fundamental knowledge and skills in elementary school social studies means knowledge for seeking to understand events in society and skills for finding and organizing information about events in society. Miyata et. al. (2017) analyzed examples of the provision of school library materials by subject and grade year. They found that in terms of quantity provided and the number of topic types in materials provided to upper grades, social studies was the most frequent. For this reason, investigative learning, which increases learners' knowledge by allowing them to search and find answers, is a particularly easy method to incorporate into elementary school social studies.

\section{Statement of the Research Problem}

Few programs focus on this structure of knowledge and aim to obtain more concrete knowledge with different contents. This study aims to develop an investigative learning support program that leads to a broad searching and understanding of subjects in social studies from multiple dimensions for elementary school students and evaluates the goal achievement of the program.

\section{Study 1}

\section{Methodology}

We searched the literature published since 2009 using the terms "school libraries" AND "classroom instruction" on CiNii Books. We analyzed a total of 21 books explaining the implementation of and instructional methods for inquiry-based learning especially at elementary schools, including the process of investigative learning and clues to set pillars.

The reason why the target was the literature published after 2009 is that in this year the report of the Children's Reading Supporters Conference was issued, and the practice of investigative and inquiry-based learning utilizing school libraries has further increased since then. In addition, since the practices of inquiry-based learning can provide suggestions for instructions and tools to 
decide sub-subjects, the practices and instructional methods of not only investigative learning but also inquiry-based learning were analyzed.

\section{Findings}

\section{Elements of the developed program}

The following elements were extracted for searching and grasping subjects from multiple dimensions in the program development: the use of image maps with terms added as clues (Horikawa \& Shioya, 2016), encyclopedias or tables of contents to expand the scope of learners' investigation (Shioya, 2014), and the incorporation of tasks for revising or adding pillars of investigation (Shioya, 2014; Tokuda, 2009).

\section{Use of image map}

As instructional methods for expanding dimensions at the planning stage, many approaches using image maps were found. An image map is a diagram created by connecting what we know and what we are interested in regarding a certain subject (Figure 2).

\section{Figure 2}

\section{Image map with clues}

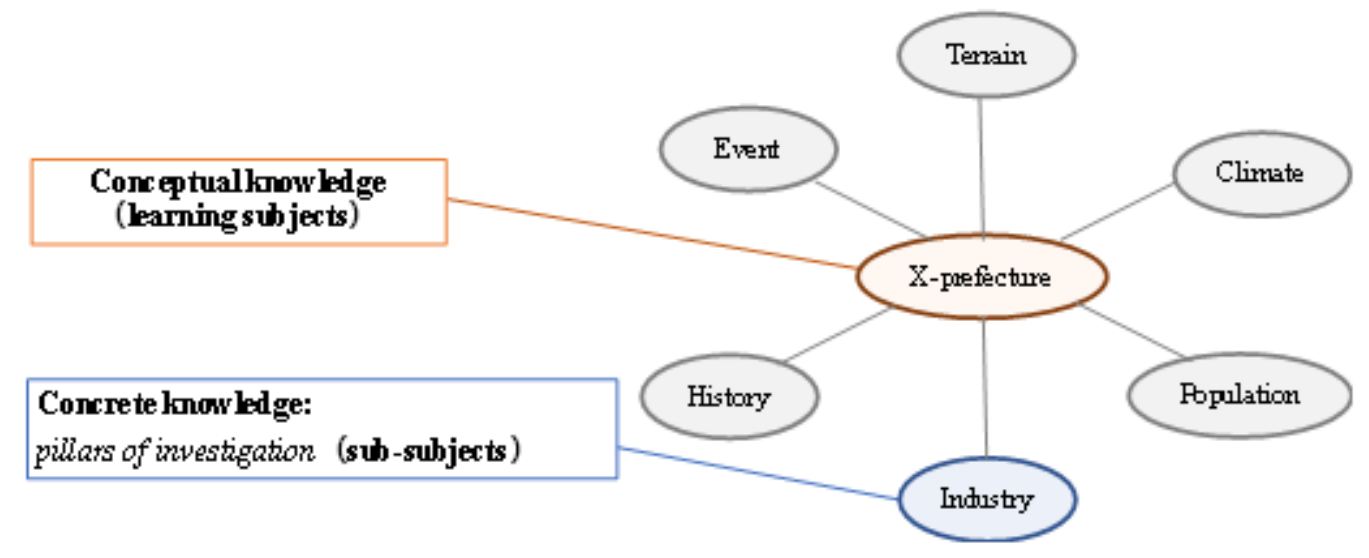

Note. Figure 2 is based on Shioya (2014).

In an image map, learners usually write down what they have come up with in relation to the subjects described in the center of the image map. Shioya (2014) pointed out that if there is little experience or knowledge about one word, the fields that can be expanded from there are limited. Shioya (2014) thus recommended that terms that can trigger the expansion should be written down in advance around the terms (subjects) that learners want to know. 
For learners to expand their own investigation, they should first use an image map with terms that correspond to concrete knowledge; in particular, there should be different content in the outer circle as clues. After that, they should move on to the next step, in which they use an image map without clues. This stepwise use of image maps is expected to lead to a broader (multidimensional) understanding of learning subjects.

\section{Use of encyclopaedias or tables of contents}

As an instructional method to expand dimensions at the planning stage (as mentioned above), an image map was found in which terms serving as clues were described in advance. However, there were no instructions regarding how the terms used as clues were determined. For learners to broaden their investigations on their own, it is necessary to experience the process of deriving clue terms and learn how to extract them.

At the information gathering stage - not at the planning stage to expand dimensions - encyclopaedias or tables of contents of books were used as a method for grasping entire learning subjects, understanding the upper and lower concepts of learning subjects, and grasping concrete knowledge with different content regarding the same learning theme (Shioya, 2014; Tokuda, 2009). The practices within the limited class time are better spent trying to establish actual concrete knowledge with different content, using encyclopaedias or tables of contents at the planning stage rather than at the information gathering stage.

\section{Incorporation of tasks for revising or adding pillars of investigation}

In the collected instructional methods, there should be an activity that involves setting up pillars of investigation once and then modifying or adding to them during the process of gathering information. For example, in Tokuda (2009), when the information was not found by using pillars of investigation set up by children at the information gathering stage, the pillars were considered inappropriate. In this case, the gathering of the information needs to be restarted with revised pillars. In addition, when searching for information and discovering other useful keywords, it is important to add new pillars. As Tokuda (2009) mentioned, the work of revising or adding these pillars of investigation approaches the solution of the problem. These additional activities are considered as important aspects of learning strategies that capture learning subjects from multiple dimensions.

\section{Overall structure of the developed program}

Since it is important to have a broad understanding of learning subjects, we determined the goals of the developed investigative learning program based on the following two points: First, it should be understood through two or more items of concrete knowledge (dimensions) with different content. Second, two or more items of concrete knowledge (dimensions) should be described at the planning stage. 
We developed the program in two steps based on the results of Study 1: Step 1: Investigative learning using the presented encyclopaedia subheadings as sub-subjects and Step 2: Investigative learning using the encyclopaedia subheadings and tables of contents of books as sub-subjects selected by learners. The units required for the program were 45 minutes in length, corresponding to one period of elementary school.

A preliminary survey was conducted at 67 public elementary schools in the Kanto region. This was designed mainly to extract learning subjects. As a result, we selected units that were implemented in many schools and contained more concrete knowledge with different content. The Step 1 and 2 units were the lives of people in distinctive regions considering the land topography and climate, and nature's perspective and the creativity and hard work of people employed in food production and the functions of transportation connecting production and consumer areas, respectively.

In addition, in the program, since children were not familiar with the term concrete knowledge, for the sake of clarity, the learning subjects that were placed at the center of the image maps were called pillars of investigation (sub-subjects).

\section{Program details}

Figure 3, Table 1 and Table 2 show the program structure of Step 1 and Step 2, respectively. Each step consists of Introduction, Expansion (main content) 1, Expansion 2, and Summary. We decided to set up a one-dimensional pillar of investigation in Expansion 1 and multiple dimensions in Expansion 2.

The structure of Expansion 1 and Expansion 2 mainly contained the following three tasks: Learners filled in sub-subjects on an image map at the planning stage, and then the information source (i.e., webpage URL and the names of school library materials) was used for gathering information for each worksheet. As the output of the investigative learning, learners were asked to describe freely what they had understood about the learning subjects.

In Summary, after comparing multiple worksheets that described what they understood by investigative learning, we decided to explain that it is important to set up pillars (sub-subjects) with different content in order to broadly understand learning subjects. In addition, we included an explanation of the importance of checking the subheadings of encyclopaedias when starting to gather information in order to provide a broader outlook. At the end, in the summary of Expansion 2, we again summarized the methods for setting up pillars of investigation.

Based on Study 1, in Step 1, learners devised a pillar of investigation based on their own ideas on image maps with clues. This was carried out to determine the dimensions from the encyclopaedia subheadings that were used as reference tools in Expansion 1 and Expansion 2. In the more 
advanced Step 2, learners reviewed the setting up of pillars based on the contents of Step 1. They first referred to encyclopaedia subheadings to construct a pillar of investigation in Expansion 1. They set a dimension on their own on an image map with no clues. Next, they referred to tables of contents of books in Expansion 2. In addition, they carried out revisions and additions to their pillars of investigation after learning how to set up multiple pillars with different content in Expansion 2.

\section{Figure 3}

\section{Program Structure}

\section{Table 1 The contents of Step 1}

\begin{tabular}{|c|c|}
\hline Introduction & 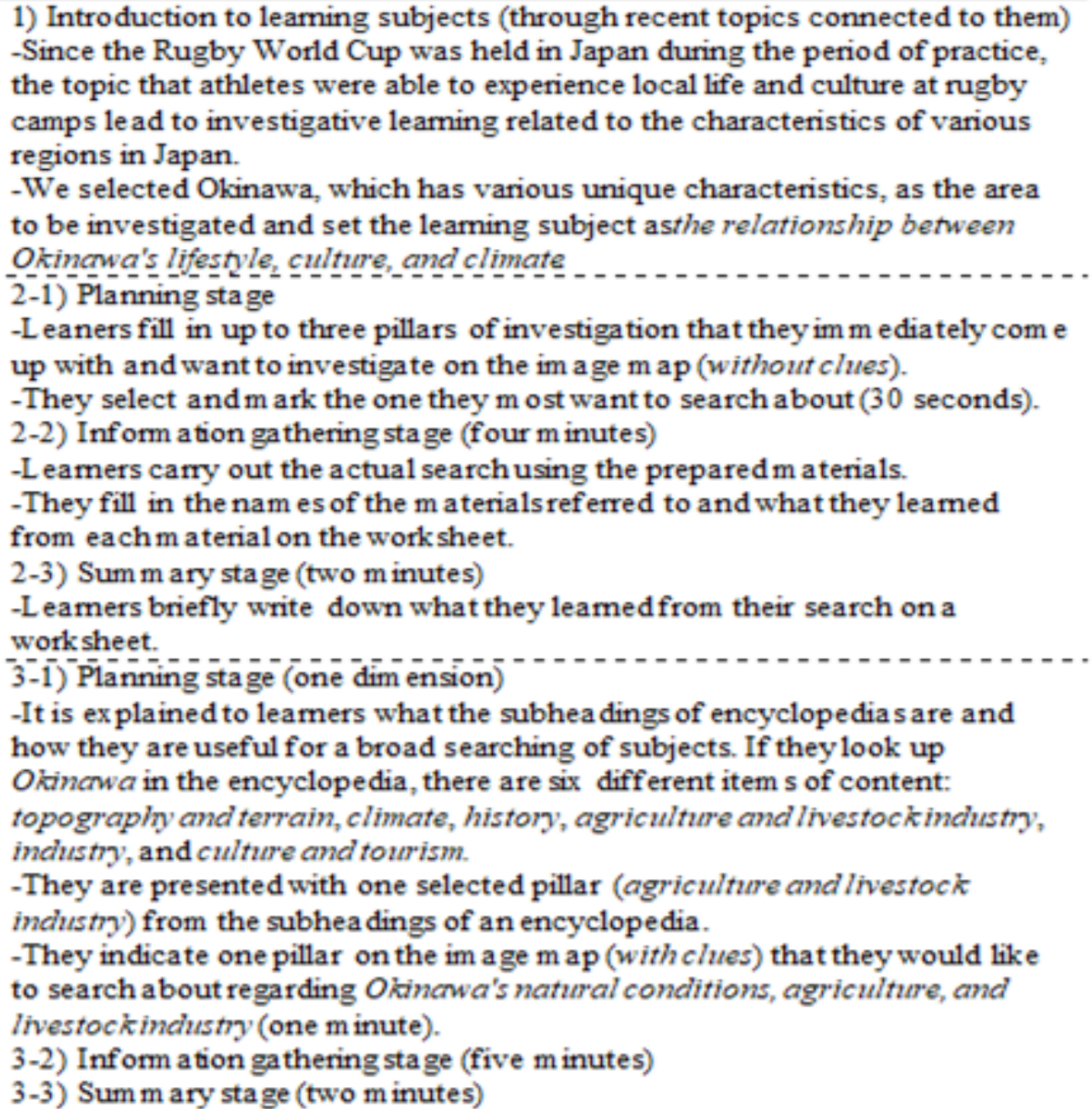 \\
\hline
\end{tabular}


Table 1 The contents of Step 1 (continued)

4-1) Planning stage (m ultiple dim ensions)
-Learners are presented with the three selected item s (agriculture and
livestockindustry, industry, and culture and tourism) from the subheadings
of the encyclopedia.
-They write down what they want to look up regarding the three pillars on the
im a ge m ap (with clues) one by one (two m inutes).
4-2) Inform ation gathering stage (six m inutes)
4-3) Sum m ary stage (two minutes)
-They refer to the pillars of investigation and write a short sum m ary of what
theylearned on the worksheet.
5) Explanation of establishing pillars of investigation at the planning stage
5-1) Comparison of three worksheets at the summary stage (2-3, $3-3$, and $4-3$ )
- Learners compare worksheet A (2-3) with B (3-3), and B (2-3) with C (3-3).
They then make a note of what they noticed.
5-2) Using the example of natural conditions and the lives of the people of
Okinawa, it is explained how to set up pillars of investigation to broadly
understand the subjects.
5-3) It is explained how looking at encyclopedia subheadings whe $\mathrm{n}$ they start
looking up something can give them a perspective that broadens their
knowledge.

Table 2 The contents of Step 2 (continued)

4-2) Modification and a ddition of im age $m$ ap

-It is ex plained that it is necessary to set up pillars (sub-subjects) with different content using the table of contents of the book.

Expansion 2

-They use the table of contents of the book to m odify the im age m ap in 4-1.

-They fill in up to five pillars with different content on the im age $m$ ap ( $n o$ clues) (three minutes).

4-3) Inform ation gathering stage (eight minutes)

4-4) Sum $m$ ary stage (three minutes)

-They refer to the pillars of investigation and write a short sum m ary of what theylearned on the work sheet.

Summary

5) Explanation of establishing pillars of investigation at the planning stage.

5-1) Comparis on of two worksheets at the summary stage (3-3 and 4-4)

- Leamers compare worksheet $\mathrm{D}(3-3)$ with $\mathrm{E}(4-4)$.

They then make a note of what they noticed.

5-2) Using the example of ingenuity and characteristics of Japanese rice cultivation, it is explained how to set up pillars of investigation to broadly understand the subjects.

5-3) It is explained that when they set up pillars of investigation from the table of contents of one book, the same chapter has similar content, so when they select pillars from different chapters, they can look up information more widely.

5-4) When setting up a pillar of investigation from the table of contents of multiple books, or when using the subheadings of encyclopedia stogether with the table s of contents of books, it is explained that they should be careful not to select pillars with similar content from chapters that are named differently but whose content is similar.

5-5) At the end, the main points are summarized. It is explained how to set up, review pillars of investigation, and gather information using pillars and summarize what they have learned. 


\section{Study 2}

\section{Methodology}

Participants

Out of fourteen students in 4th-6th grades who attended public primary schools in the Kanto region, we evaluated a total of six students who completed all their assignments.

\section{Preparation for the program}

From the book lists published by the prefectural library that has actively supported school libraries, nine and twelve books were selected as materials for Steps 1 and 2, respectively.

\section{Procedure}

The program was put into practice and evaluated over four weeks. Step 1 was conducted in the second week and Step 2 in the third week. Each step lasted 45 minutes.

In the first and fourth weeks, take-home assignments were given to examine the goal attainment of the program. The assignments consisted of a student independently using a worksheet to conduct each step of the investigative learning (pillars of investigation, searching for information, and summarizing). Learners first selected one area from the list of official Rugby World Cup campsites, wrote freely on the worksheet what they already knew before searching, and then filled in the pillars (sub-subjects) on the image map with five outer circles without clues. Next, they recorded on the worksheet the information source (webpage URL and names of books) that they actually referred to and what information they obtained from each source. Finally, they wrote down on the worksheet what they learned through searching.

In the first week, we conducted a questionnaire on the frequency of investigative learning in school classes. The students were asked to choose one on a scale of a to i:

a. at least once a week,

b. once a week,

c. once every two weeks,

d. once a month,

e. once every two or three months,

f. once every six months,

g. once a year,

h. less than once a year,

i. Do not experience at all.

We paid a reward to the participants who completed all their assignments in four weeks in accordance with university regulations. This study was conducted with the approval of the research ethics review committee. 


\section{Evaluation method}

Based on the results of the preliminary survey, various industrial production and distribution of areas in Japan was selected as the subject of the take-home assignments.

The first and fourth weeks involved a unit related to industry, and the evaluation criteria (concrete knowledge) were the following five items based on the explanations provided by the Course of Study: type of industry, production value by type, number and ratio by factory size, Japan's industrial zones, and distribution of industrial zones. The results of the sub-subjects on the image map and the free descriptions in the worksheets were classified according to the concrete knowledge related to the unit. The number of items of concrete knowledge were counted for scoring.

The planning and understanding of subjects from multiple dimensions were measured using the following five levels: zero points had no dimension; one point had a single dimension; both two and three points had two dimensions, but two points established pillars of investigation for similar contents with a single dimension, and three points established pillars of investigation for different contents with two dimensions; and finally, four points had more than two dimensions. The study determined that learners with three points or more achieved the goal.

\section{Findings}

\section{Progress of the program}

There was no major confusion in terms of the progress of the program, and we were able to proceed with the developed program almost as planned.

\section{Investigative learning experience}

Five out of six students experienced investigative learning at least once a week, and only one studied once every two weeks.

\section{Goal evaluation of the program}

As a result, three out of six students achieved the goals after the program was implemented. None of the students had a reduced level with regard to planning or understanding. The students who achieved the goal for planning also achieved the goal for level of understanding.

With regard to the three students who could not achieve the goal, two students set up multiple pillars of investigation of the subjects. However, one of them had similar pillars. The other student set up the pillars related not to the learning subjects. Another student, who had less experience in investigative learning, had only one pillar of investigation.

However, half of the children did not meet the achievement goals. None of the children who did not achieve the goal of understanding did not achieve the goal of planning. They had similar 
pillars even if they had filled in multiple pillars or only one pillar. In addition, they selected pillars related not to industry but to other industries or crafts. As a result of this, we suggest that it is necessary, especially for elementary school students, to first confirm the differences between terms similar to the learning subjects and the original learning subjects.

\section{General Discussion}

This study aimed to develop an investigative learning support program that would lead to broad searching and understanding of subjects in social studies from multiple dimensions for elementary school students and evaluate the goal achievement of the program.

We developed an investigative learning support program consisting of two steps with multiple features including pillars of investigation. We used reference materials and image maps, both with and without clues, and incorporated the tasks of revising or adding pillars of investigation.

The program contained two steps:

Step 1: Investigative learning using the presented encyclopaedia subheadings as sub-subjects Step 2: Investigative learning using the encyclopaedia subheadings and tables of contents of books as sub-subjects selected by learners.

The goals of the developed investigative learning program were evaluated with respect to whether children could plan for and understand learning subjects with two or more items of concrete knowledge (dimensions) with different content.

The evaluation of the program practice showed that half of the children met the program achievement goals, and no children had a decrease with regard to their level of planning or understanding. All children who met the program's goals were provided during the planning stage with perspectives related to concrete knowledge with different content.

However, half of the children did not meet the achievement goals. None of the children who did not achieve the goal of understanding did not achieve the goal of planning. They had similar pillars even if they had filled in multiple pillars or only one pillar. In addition, they selected pillars related not to industry but to other industries or crafts. As a result of this, we suggest that it is necessary, especially for elementary school students, to first confirm the differences between terms similar to the learning subjects and the original learning subjects.

One child who did not meet the achievement goals had little experience in investigative learning and stumbled at the planning stage and the information gathering stage. This result was consistent with Shioya's (2014) suggestions. In the future, it is important to consider how to deal with children who have little experience in investigative learning. 


\section{Implications and Conclusions}

In this study, a two-step investigative learning support program was developed:

1. Investigative learning using the presented encyclopaedia subheadings as sub-subjects

2. Investigative learning using the encyclopaedia subheadings and tables of contents of books as sub-subjects selected by learners.

The goals of the program were determined as the following two points: understanding and planning through two or more items of concrete knowledge (dimensions) with different content.

We conducted this program and evaluated the goal achievement of the program. Some students meet the achievement goals. However, issues were also posed in relation to supporting students who have little experience in investigative learning, and suggestions for enabling them to accurately grasp learning subjects were provided.

In the future, it will be necessary to increase the number of participants in the practice and further examine the goal achievement of the revised program, and its application in other subjects.

\section{References}

Horikawa, T. \& Shioya, K. (2016). Gakushuusidou to gakkoutoshokan (Instruction for Learning in School Library). Housoudaigakukenkyuushinkoukai (Foundation for the Promotion of the Open University of Japan)

Kuhlthau, C. C., Maniotes, L. K., \& Caspari, A. K. (2015). Guided inquiry: Learning in the 21st century, 2nd ed. Libraries Unlimited.

Kuwata, T. (2016). Shikou wo fukameru tankyuu gakushuu: akutibu râningu no shiten de katsuyou suru gakkou toshokan (Inquiry-based learning to deepen understanding: School library utilized from a viewpoint of active learning) . Zenkokugakkoutoshokankyougikai (Japan School Library Association)

Ministry of Education, Culture, Sports, Science and Technology (2017).

Gakushuushidouyouryou (Courses of Study). Retrieved from

https:/www.mext.go.jp/a_menu/shotou/new-cs/1383986.htm

Miyata, R., Tada, S., \& Asaishi, T. (2017). Analysing school librarians' practices in providing useful materials for classes to support teachers. Journal of Japan Society of Library and Information Science, 64, 115-131.

Shioya, K. (2014). Tankyuutekina gakushuu wo sasaeru: Tsukamu, Sagasu, Erabu, Matomeru (Information literacy skills that support inquiry-based learning: Grasp, search, select, and summarize) Zenkokugakkoutoshokankyougikai (Japan School Library Association)

Tokuda, E. (2009). Shougakkou ni okeru manabikata no shidou: Tankyugakushuu wo susumeru tameni (Teaching how to learn in elementary school: To promote inquiry learning) Zenkokugakkoutoshokankyougikai (Japan School Library Association)

Tokyo Shoseki (2015). Heisei 27 nendoyou shinpen Atarashii shakai nenkanshidoukeikakusakuseisiryou “Chisiki no kouzouzu 5 nen jyoukan” (New edition 
for 2015 school year, A material for new social studies annual guidance plan "Structure of knowledge for fifth grade, first volume") Tokyo Shoseki 